\title{
Indicadores de desempeño ambiental en el proceso de abastecimiento de la industria de plástico
}

\author{
Emilia Avilés-Sánchez* \\ Fernando Lámbarry-Vilchis**
}

Fecha de recibido: 14 de marzo de 2021

Fecha de aprobado: 20 de octubre de 2021

\begin{abstract}
Para citar este artículo: Avilés-Sánchez, E., \& Lámbarry-Vilchis, F. (2022). Indicadores de desempeño ambiental en el proceso de abastecimiento de la industria de plástico. Revista Universidad \& Empresa, 24(42), 1-25. https://doi.org/10.12804/revistas.urosario. edu.co/empresa/a.10381
\end{abstract}

\section{Resumen}

El objetivo de la investigación es proponer indicadores de desempeño ambiental para el proceso de abastecimiento en las industrias medianas del plástico. Estas empresas son las de mayor dinamismo en cuanto a su producción, pero también en cuanto a contaminación y, aunque en la literatura se encuentran aproximaciones hacia su sustentabilidad, para la etapa de abastecimiento son escasos o inexistentes. Los resultados muestran que los indicadores deben establecerse desde el subproceso de proveedurías para minimizar los impactos financieros derivados del retrabajo, rechazos de materiales u omisiones de actividades en el proceso.

Palabras clave: indicadores ambientales; proceso de abastecimiento; proceso de abastecimiento industria del plástico; indicadores de desempeño ambiental.

\footnotetext{
* Profesora-Investigadora del Instituto Politécnico Nacional. ESCA Santo Tomás. Correo electrónico: eaviles@ipn.mx ORCID: https://orcid.org/0000-0002-0871-9621

** Profesor-Investigador del Instituto Politécnico Nacional. ESCA Santo Tomás. Correo electrónico: flambarry@ipn.mx ORCID: https://orcid.org/0000-0002-0216-1647
} 


\title{
Environmental Performance Indicators for Procurement in Plastics Industries
}

\begin{abstract}
The objective of this research was to propose environmental performance indicators for the procurement in medium-sized plastics industries. These companies are the most dynamic in terms of their production, but also in terms of contamination and although in the literature there are approaches towards their sustainability, for the procurement they are scarce or non-existent. The results show that the indicators must be established from the sub-process of suppliers to minimize the financial impacts derived from rework, rejection of materials or omissions of activities in the process.
\end{abstract}

Keywords: Environmental indicators; procurement; purchasing process of plastic industry; environmental performance indicators.

\section{Indicadores de desempenho ambiental no processo de abastecimento da indústria de plásticos}

\section{Resumo}

O objetivo da pesquisa foi propor indicadores de desempenho ambiental para o processo de abastecimento em indústrias de plásticos de médio porte. Estas empresas são as mais dinâmicas em termos de produção, mas também em termos de poluição e embora na literatura existam abordagens para a sua sustentabilidade, para a fase de abastecimento tais abordagens são escassas ou inexistentes. Os resultados mostram que os indicadores devem ser estabelecidos a partir do subprocesso de fornecedores para minimizar os impactos financeiros decorrentes de retrabalho, rejeição de materiais ou omissões de atividades no processo.

Palavras-chave: indicadores ambientais; processo de abastecimento; processo de abastecimento da indústria de plástico; indicadores de desempenho ambiental.

\section{Introducción}

La industria del plástico es una de las más importantes en el mundo, participa con el 56\% del producto interno bruto en la Unión Europea, mientras que en América Latina con el 25\% (Comisión Económica para América Latina y el Caribe [CEPAL], 2018; Dini \& Stumpo, 2011; Giosa Zuazua \& Fernández Massi, 2017). Además, por el dinamismo y ubicuidad, se ha convertido en uno de los materiales de uso más común en la economía global, con una producción que se estima de 407 Millones de toneladas por año (Mtpa), pero con un proceso de transformación energético-intenso que conlleva 400 millones de toneladas de emisiones de efecto invernadero (Organización para la Cooperación y el Desarrollo, OECD, 2018), además de diversos impactos ambientales asociados con su deficiente gestión 
y disposición de sus residuos con tasas bajas de solo 9\% de reciclaje (oECD, 2018). Consecuente con ello, se ha propuesto en la literatura especializada abordar el impacto ambiental de sus procesos, desde la arista de la sustentabilidad y más recientemente de la circularidad de las organizaciones; uno de ellos de creciente interés es en su proceso de abastecimiento (Farooque et al., 2019; Walker et al., 2012), que se ha conceptualizado en la literatura especializada indistintamente bajo diferentes formas, tales como cadenas de suministro sustentables (Meherishi et al., 2019; Sönnichsen \& Clement, 2020), cadenas de suministro verdes (Asif et al., 2020; Malviya \& Kant, 2017) o cadenas de suministro circulares (Farooque et al., 2019; Lahane et al., 2020).

Pese a esta diversidad conceptual, son coincidentes en que la sustentabilidad debe integrarse en las primeras etapas del abastecimiento y considerarse cuidadosamente durante todo el proceso (Appiagyei et al., 2016). Es por ello que el proceso de abastecimiento se encuentra en una posición central en el desarrollo de una cadena de suministro y organización sustentable. Derivado de esto, un primer paso para alcanzar la sustentabilidad del proceso es la identificación de indicadores de impacto ambiental, que minimicen el uso de energía, reduzcan el ruido y la contaminación, y hagan eficiente el manejo de los recursos y la producción (Appiagyei et al., 2016; Kalubanga, 2012). Sin embargo, los estudios sobre este tema son escasos, algunos de ellos se orientan más a la gestión sustentable del proceso de compras (Schneider \& Wallenburg, 2012), al proceso de abastecimiento social y ambientalmente responsable (Hoejmose \& Adrien-Kirby, 2012), a la valoración de eficiencia sustentable de los proveedores (Giannakis et al., 2020) e incluso a las barreras de su adopción en las organizaciones (Bai \& Satir, 2020; Giunipero et al., 2012), a pesar de que se cuenta con las series de la Organización Internacional de Estandarización (Iso por sus sigla en inglés) ISO 20400:2017, ISO 14000, ISO 26000:2010 y el Sistema Comunitario de Ecogestión y Auditoría Medioambiental vinculados con la sustentabilidad en el proceso de compras en las organizaciones, pero que no han sido estudiados a fondo.

Por lo tanto, esta investigación de corte documental plantea como objetivo proponer indicadores clave de rendimiento e impacto ambiental para el proceso de abastecimiento en las empresas medianas de plástico. 


\section{Proceso de abastecimiento en la mediana industria del plástico}

El proceso de abastecimiento de materia prima comprende el ciclo completo de identificación de necesidades (adquisición de bienes o servicios de un proveedor) hasta el contrato de servicios o, bien, del final de la vida útil de los bienes, incluida la gestión de desperdicios (Iso, 2017). En este sentido, los procesos describen las actividades concernientes al suministro e identifican los impactos negativos, desde la fuente de abastecimiento, los materiales renovables o no renovables, reciclados o vírgenes, hasta la relación comercial con el proveedor y la implementación de mecanismos de respuesta formal de quejas o reclamaciones, incluyendo responsabilidad en las interrupciones en el proceso o sugerencias de innovación en el mismo (Global Sustainability Standards Board, GssB, 2016d, 2016e, 2016g, 2016h, 2016f; Iso, 2017). Rivera, Cristobal y Aguilar (Rivera González et al., 2011), al igual que el modelo de Referencia de Operaciones de la Cadena de Suministro (scor por sus siglas en ingles), basan la gestión de compra con relación a la naturaleza de los insumos, la ejecución de actividades con el almacén o procesos internos, la identificación de proveedores mediante la actividad principal del responsable de adquisiciones para el desarrollo de estrategias y la administración del contrato con proveedores, como parte de las actividades externas y soporte de la organización (Bolstorff \& Rosenbaum, 2011).

Así mismo, Krajewski et al. (2013) y Cabeza (2014) basan la integración del proceso de abastecimiento en vínculos funcionales y organizacionales internos y externos, resultado de acciones estratégicas realizadas con los proveedores. Por otro lado, Chopra y Meindl (2013), y Ruiz Torres et al. (2013) avalan que el proceso está relacionado con la planeación, implementación y el control eficiente del flujo efectivo de costos, así como del almacenaje correcto de materia prima, la administración del material intermedio o de proceso, y del inventario de producto final, los cuales se reflejan en: 1) instalaciones, 2) inventario o programación de compras de materia prima, 3) la recepción del material en la planta y transporte, 4) información, 5) abastecimiento en control de calidad del material para aceptación o rechazo de material y 6) fijación de precios. En tanto que Mercado (2002, 2009) y Paesbrugghe et al. (2017) coinciden en que el proceso de suministro se compone de actividades de servicios proporcionados y enfocados al proveedor, como la búsqueda 
de nuevas fuentes para la adquisición de materia prima, costos, precio y acuerdos de servicio enfocados a consultoría para el aprovechamiento de las oportunidades y solución de problemas presentados en el suministro, soporte en la información y actividades a la organización, certificaciones y normas gubernamentales, así como la responsabilidad para la colaboración en procesos para la innovación.

Aunque también se tiene la percepción de que el proceso de abastecimiento se divide en tres subprocesos: actividades con el proveedor, responsabilidad del personal de abastecimiento, y procesos internos (Bolstorff \& Rosenbaum, 2011; Chopra \& Meindl, 2013; GssB, 2016e; ISO 20400:2017(E), 2017; Krajewski et al., 2013) de manera que cada uno de ellos es complementario del otro, en la identificación de los riesgos asociados con el desempeño ambiental que parte desde la búsqueda de proveedores hasta la negociación de la materia prima (Chopra \& Meindl, 2013; Krajewski et al., 2013; Mercado, 2002), en la planificación operativa y en la gestión de las actividades del proceso (Bolstorff \& Rosenbaum, 2011; Global Sustainability Standards Board (GssB), 2016f, 2016i; ISO 20400:2017(E), 2017; Ruiz Torres et al., 2013), además de la validación de las actividades internas en la mejora del abastecimiento y por las de valor agregado reflejadas en los precios, costos, calidad de las operaciones y la disminución de material rechazado (Chopra \& Meindl, 2013; ISO 14001:2015(E), 2015a; ISO 9001:2015, 2015; Krajewski et al., 2013; Mercado, 2002; Paesbrugghe et al., 2017).

\section{Desempeño e impacto ambiental en el proceso de abastecimiento: revisión teórica}

La cadena de suministro sustentable se define como "las adquisiciones que tienen impacto ambiental, social y económico más positivos posibles a lo largo de todo el ciclo de vida y que se esfuerzan por minimizar los impactos adversos" (Iso, 2017). Derivado de ello, una parte de la literatura reciente destaca la inclusión de factores ambientales y sociales por encima de los económicos en el proceso de compra de la organización (Crespin-Mazet \& Dontenwill, 2012), planteando la necesidad de establecer, más allá de las financieras, nuevas métricas de impacto ambiental (Carter \& Rogers, 2008). 
Además de una creciente conciencia de los consumidores sobre las emisiones contaminantes, el consumo de agua y energía durante el ciclo de vida del producto que adquieren ha presionado para que inicialmente en las cadenas de suministro se empiece a considerar que se cumplan los criterios ambientales para minimizar su impacto (Appiagyei et al., 2016). Ejemplos muy recurrentes son: la incorporación de criterios de desempeño ambiental en las etapas de selección y la evaluación de proveedores durante el proceso de abastecimiento (Crespin-Mazet \& Dontenwill, 2012; Kalubanga, 2012), las empresas están priorizando a los proveedores más considerados con el medio ambiente. Es una práctica naciente en países en desarrollo (Kalubanga, 2012) y más aún en la industria del plástico, en la que el proceso de suministro de materias primas está enfocado, principalmente, en la gestión de desempeño por resultados en cuanto a los costos, ventas, productividad y gastos (Aureli et al., 2018; Balfaqih et al., 2016; Forkmann et al., 2016; Ganguly \& Shankar Rai, 2018; Graça \& Camarinha-Matos, 2017; Iso, 2015b; Iso, 2015), al reciclaje (Borkey \& Leinala, 2018; Castelan, 2018; Horvath et al., 2018; Mathiyazhagan et al., 2016) y escasamente a los indicadores de impacto ambiental (Giunipero et al., 2012; Kipp et al., 2012; Mura et al., 2018; Samaniego \& Schneider, 2010; Sundarakani et al., 2008).

No obstante, existen modelos internacionales fundamentados en la serie International Standarization Organization ISO 20400:2017, ISO 14000, ISO 26000:2010 o en el Sistema Comunitario de Gestión y Auditoría Medioambientales (Eco-Management and Audit Scheme, EMAs), que proponen indicadores generales de desempeño ambiental (IDA) e indicadores de condición ambiental (ICA), con la flexibilidad de adaptarse para el proceso de abastecimiento (anexo 1); algunos orientados a la destrucción de la flora y fauna como parte de la norma (Dragomir, 2018; EMAs, 2013; ISO, 2015b; ISO, 2016) pero no como parte del proceso; otros modelos más al impacto por actividades en el cambio climático o por inventarios, rutina de actividades y servicio en el proceso (Gssb, 2016d, 2016g, 2016f, 2016h; López Blanco \& Rodríguez Gamiño, 2009; López López, 2017; Sánchez López \& Meza Arístigue, 2010); o bien en la identificación de ingresos, gastos corrientes e inversiones o ingresos por disposición y venta de residuos y desperdicios (Castelan, 2018; Cervera-ferri \& Ureña, 2017; Dragomir, 2018; Manteiga, 2000); hacia aspectos de conformidad por el servicio de proveedores como respuesta a incidentes que impactan en el ambiente, o relacionados con la obtención de materia prima, cambio de patrones de consumo, la respuesta en cuanto al material rechazado de productos de insumos o productos finales, e incluso 
los relacionados con pérdidas o desastres naturales (Castelan, 2018; Cervera-ferri \& Ureña, 2017; Dragomir, 2018; ISO, 2015b; ISO, 2016; ISO, 2015; Manteiga, 2000), otros modelos más en la relación que tienen los costos financieros ambientales como inversiones o gastos en la protección ambiental y la identificación de actividades contaminantes durante los procesos (EMAS, 2013; ISO, 2015b; Chávez, 2005); unos más enfocados a la integridad en la transparencia de los procesos y la recurrente capacitación para la integración del impacto ambiental en el desarrollo de toma de decisiones para el cambio de pensamiento hacia el medio ambiente (GSSB, 2016a, 2016b, 2016c, 2016d, 2016e, 2016f, 2016g, 2016h, 2016i, 2016j; López López, 2017; OIT, 2018; Sánchez López \& Meza Arístigue, 2010).

\section{Método de investigación}

El objetivo de esta investigación es proponer indicadores de impacto ambiental para el proceso de abastecimiento de las empresas medianas de plásticos; para ello, el método consistió en dos fases principales.

Una primera de mapeo del proceso de abastecimiento, la cual se realizó a través de un análisis documental; se seleccionaron aquellos subprocesos y actividades que fueron los más recurrentes en los propuestos por Bolstorff y Rosenbaum (2011), Cabeza (2014), Chopra y Meindl (2013), GssB (2016d, 2016g, 2016h, 2016f, 2016e), Iso (2017), Krajewski et al. (2013), Mercado (2002, 2009), Paesbrugghe et al. (2017), Rivera et al. (2011), y Ruiz Torres et al. (2013). La selección de estos modelos se fundamentó en la popularidad y por ser los más documentados en la literatura sobre procesos de abastecimiento, con la posibilidad de adaptarse a la industria del plástico. En esta misma fase se seleccionaron los indicadores ambientales que aplicaran al proceso de suministro de 15 modelos encontrados en la literatura especializada: 1. ISO14001 (ISO, 2015a); 2. ISO9001 (ISO, 2015); 3. ISO26000 (ISO, 2010); 4. Isus Indicadores de sustentabilidad (López López, 2017); 5. Іамв Indicadores ambientales para países de América Latina y el Caribe (Martínez, 2009); 6. IINv Indicadores de iniciativa de industria verde (Martínez et al. 2009); 7. sIPv Sistema de Indicadores de Producción Verde (Cervera-ferri \& Ureña, 2017); 8. IDSM Indicadores ambientales y de sustentabilidad en México (Instituto Nacional de Estadística, Geografía e Informática, 1999); 
López Blanco \& Rodríguez Gamiño, 2009); 9. ods Objetivos de desarrollo sostenible (onu, 2017); 10. CEMDs Consejo Empresarial Mundial para el Desarrollo Sostenible (Martínez, 2009); 11. IEMPV Indicadores de empleos verdes de la Organización Internacional del Trabajo (OIT, 2018); 12. IIAME Indicadores de impacto ambiental para empresas medianas (Dragomir, 2018; Briggs, 2017); 13. IEMAs Indicadores medioambientales para la Industria Española (EMAS, 2013; IHOBE, 1999); 14. IRES Indicadores de responsabilidad social CEMEFI (Calderón, 2012); 15. IGRI Indicadores para el reporte global de sustentabilidad (GssB, 2016b, 2016a, 2016c).

La segunda fase consistió en la validación, realizada con siete expertos laboralmente activos en la gestión de procesos de abastecimiento de medianas industrias de plástico de la Ciudad de México, de los subprocesos, actividades e indicadores de impacto ambiental, propuestos para la cadena de suministro. Las entrevistas se realizaron en forma electrónica del 17 de octubre al 21 de noviembre de 2019.

\section{Resultados y discusión}

\subsection{Primera fase}

El proceso de suministro para la industria mediana de plástico resultante se divide en tres subprocesos (figura 1): 1. Proveedor, con actividades que implican la colaboración, selección y negociación; 2. Responsabilidad del personal de abastecimiento, relacionado con la búsqueda de proveedores, la gestión y emisión de órdenes de compra, la planeación y establecimiento de parámetros de compra, la eliminación de reprocesos y costos ambientales; y 3. Procesos internos, con actividades de recepción y rechazo de material y reciclaje. 


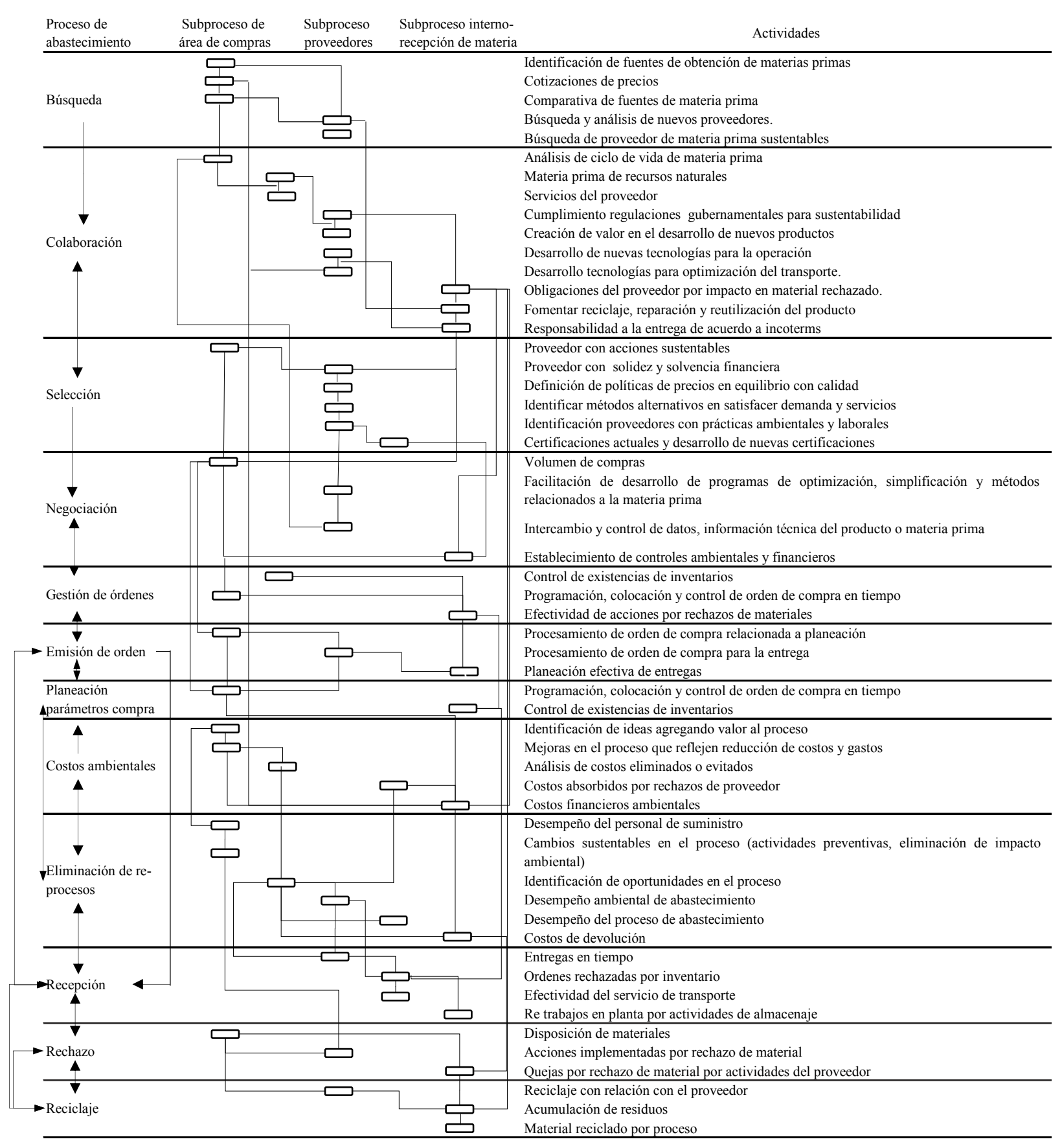

Figura 1. Proceso de abastecimiento de la mediana industria del plástico

Fuente: elaboración propia a partir de Bolstorff y Rosenbaum (2011); Cabeza (2014); Chopra y Meindl (2013); GSSB (2016d, 2016g, 2016h, 2016f, 2016e); ISO (2017); Krajewski et al. (2013); (Martínez et al. 2009); Mercado (2002, 2009); Paesbrugghe et al. (2017); Rivera González et al. (2011); Ruiz Torres et al. (2013) y retroalimentación de siete expertos. 


\subsection{Segunda fase}

De los 15 modelos seleccionados es perceptible que basan sus indicadores en la gestión de residuos o en la huella de carbono que se genera en la totalidad del proceso y no en el desempeño propio de cada una de las actividades del proceso, en este caso de abastecimiento. Se analizaron los modelos y resultaron 48 categorías que agrupaban a los indicadores de desempeño ambiental, no obstante, algunas de ellas similares en nombre se redujeron y, en algunos casos, se reagruparon en categorías más amplias, pero cuidando que fueran representativas del concepto al que aludían (anexo 1). Fueron 13 las validadas por retroalimentación del grupo de expertos, las cuales son aplicables al proceso de abastecimiento (tabla 1). Este proceso de validación implicó la elección, por parte de los siete expertos, de las categorías más importantes que impactaran el desempeño ambiental del proceso de compra, finalmente, como criterio de decisión se seleccionaron aquellas que al menos fueran seleccionadas por mayoría relativa (tres o más) de los expertos.

Los indicadores inician desde la identificación de la búsqueda de nuevas fuentes de materia prima, lo que permite una transición a procesos y certificaciones sustentables, independientemente de la madurez de la relación comercial con el proveedor. Así mismo, impactan en diferentes subprocesos del suministro y representan bajo este enfoque una medición del desempeño del proveedor y del responsable del puesto en la recepción de material, de esta manera se promueve una correlación entre la gestión y los resultados de la operación.

Los indicadores que parten de las certificaciones, por parte de la proveeduría con el medio ambiente (cumplimiento de normas legales medio ambientales), continúan con las métricas de emisiones que resultan del proceso de las actividades (emisiones de gases de efecto invernadero al ambiente), la concientización hacia el medio ambiente de las personas vinculadas a la organización (empleo verde y capacitación en procesos verde), la identificación y medición de residuos (generación de residuos), el impacto ambiental por el desempeño del responsable del proceso (rutina de actividades en el proceso e impacto ambiental por uso de instalaciones y equipos), la correcta gestión para la disposición de material peligroso (materiales peligrosos y dañinos al ambiente), el uso de servicios de proveedores de materias primas, que además dan soporte a la organización para la disminución del impacto ambiental (uso de materias primas sustentables, cantidad de 
materias primas no renovables, proveedores con certificaciones para la sostenibilidad y proveedores con servicios de soporte a las operaciones de la organización), de manera que, con la gestión correcta de los procesos se pueda identificar acertadamente el costo generado por el impacto ambiental, por la gestión y la toma de decisiones, basada en riesgo (transferencia de costos ambientales), y por la transparencia de las operaciones mediante la ética en el proceso (integridad y procesos operativos justos) aplicados de acuerdo a cada rango y parámetro del proceso de suministro (figura 2).

Tabla 1. Matriz de relación de indicadores de desempeño de impacto ambiental, con las fases del proceso de abastecimiento

\begin{tabular}{|c|c|c|c|c|c|c|c|c|c|c|c|c|}
\hline 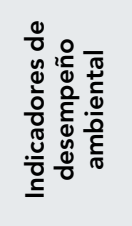 & 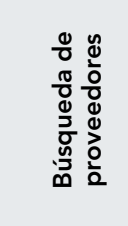 & 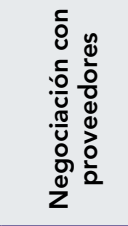 & 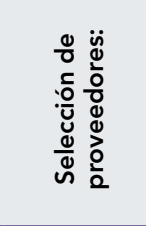 & 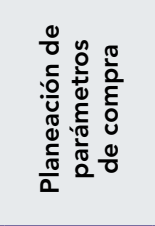 & 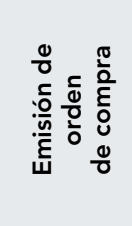 & 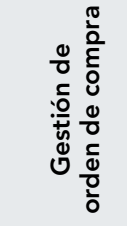 & 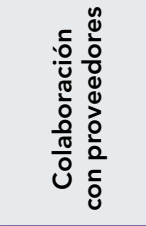 & 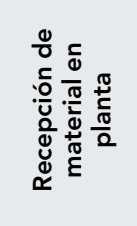 & 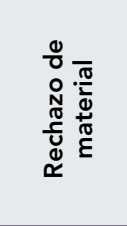 & 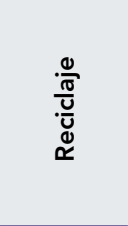 & 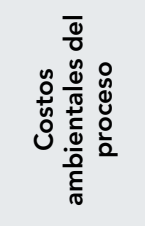 & 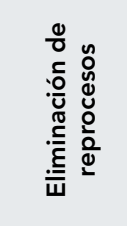 \\
\hline $\begin{array}{l}\text { Cumpli- } \\
\text { miento de } \\
\text { normas } \\
\text { legales } \\
\text { medio } \\
\text { ambiente. }\end{array}$ & $\begin{array}{l}\text { ISO14-9- 26, } \\
\text { IRES, } \\
\text { IGRI }\end{array}$ & & $\begin{array}{l}\text { ISO14-9-26, } \\
\text { IRES,IGRI }\end{array}$ & & & & $\begin{array}{l}\text { ISO14-9- } \\
\text { 26,IRES,IGRI }\end{array}$ & & & & $\begin{array}{l}\text { ISO14-9-26, } \\
\text { IIAME,IRES }\end{array}$ & \\
\hline $\begin{array}{l}\text { Emisiones } \\
\text { de gas de } \\
\text { efecto } \\
\text { invernadero }\end{array}$ & & & $\begin{array}{l}\text { ISO14-9-26, } \\
\text { ISUS,IAMB, } \\
\text { IINV,SIPV, } \\
\text { IDSM,ODS, } \\
\text { CEMDS, } \\
\text { IEMPV, IIAME, } \\
\text { IEMAS, } \\
\text { IRES,IGR }\end{array}$ & & $\begin{array}{l}\text { ISO14-9-26, } \\
\text { ISUS,IAMB, } \\
\text { IINV,SIPV, } \\
\text { IDSM,ODS, } \\
\text { IIAME, } \\
\text { IEMAS,IRES, } \\
\text { IGRI }\end{array}$ & $\begin{array}{l}\text { ISO14-9-26, } \\
\text { ISUS,IAMB, } \\
\text { IINV,SIPV, } \\
\text { IDSM, } \\
\text { CEMDS, } \\
\text { IIAME, } \\
\text { IEMAS, } \\
\text { IRES,IGRI }\end{array}$ & $\begin{array}{l}\text { ISO14-9-26, } \\
\text { IAMB,IINV, } \\
\text { IDSM, } \\
\text { CEMDS, } \\
\text { IEMPV, } \\
\text { IEMAS,IRES, } \\
\text { IGRI }\end{array}$ & $\begin{array}{l}\text { ISO14-9-26, } \\
\text { ISUS,ODS, } \\
\text { CEMDS, } \\
\text { IEMPV, } \\
\text { IIAME, } \\
\text { IEMAS, } \\
\text { IRES,IGR }\end{array}$ & $\begin{array}{l}\text { ISO14-9-26, } \\
\text { ISUS,IAMB, } \\
\text { IINV,SIPV, } \\
\text { IDSM, } \\
\text { CEMDS, } \\
\text { IEMPV, } \\
\text { IIAME, } \\
\text { IEMAS,IGRI }\end{array}$ & $\begin{array}{l}\text { ISO14-9-26, } \\
\text { ISUS,SIPV, } \\
\text { IDSM, } \\
\text { CEMDS, } \\
\text { IIAME, } \\
\text { IEMAS, } \\
\text { IRES,IGRI }\end{array}$ & & \\
\hline $\begin{array}{l}\text { Empleo } \\
\text { verdey } \\
\text { capacitación } \\
\text { en procesos } \\
\text { verde }\end{array}$ & & & IGRI & $\begin{array}{l}\text { ISO14-9-26, } \\
\text { IIAME }\end{array}$ & & $\begin{array}{l}\text { ISO14-9-26, } \\
\text { IGRI }\end{array}$ & & IGRI & ISO14-9,26 & & & ISUS,IGRI \\
\hline $\begin{array}{l}\text { Generación } \\
\text { de residuos }\end{array}$ & & & & $\begin{array}{l}\text { ISO14-9-26, } \\
\text { ISUS,IAMB, } \\
\text { IDSM, } \\
\text { CEMDS, } \\
\text { IEMAS,IRES, } \\
\text { IGR }\end{array}$ & & & $\begin{array}{l}\text { ISO14-9-26, } \\
\text { ISUS,SIPV, } \\
\text { IDSM,ODS, } \\
\text { IIAME,IEMAS, } \\
\text { IRES }\end{array}$ & $\begin{array}{l}\text { ISO14-9-26, } \\
\text { ISUS,SIPV, } \\
\text { IDSM,ODS, } \\
\text { CEMDS, } \\
\text { IRES,IGRI }\end{array}$ & $\begin{array}{l}\text { ISO14-9-26, } \\
\text { ISUS,IAMB, } \\
\text { SIPV,IDSM, } \\
\text { ODS,IIAME, } \\
\text { IEMAS }\end{array}$ & $\begin{array}{l}\text { ISO14-9-26, } \\
\text { ISUS,IAMB, } \\
\text { IDSM, } \\
\text { CEMDS, } \\
\text { IEMPV, } \\
\text { IIAME,IRES }\end{array}$ & $\begin{array}{l}\text { ISO14-9-26, } \\
\text { ISUS,IAMB, } \\
\text { IDSM, } \\
\text { CEMDS, } \\
\text { IEMAS, } \\
\text { IRES,IGRI }\end{array}$ & \\
\hline $\begin{array}{l}\text { Impacto } \\
\text { ambiental } \\
\text { por rutina } \\
\text { de activi- } \\
\text { dades en el } \\
\text { proceso }\end{array}$ & & IGRI & & $\begin{array}{l}\text { ISO14-9-26, } \\
\text { ISUS,SIPV, } \\
\text { IDSM,CEMDS, } \\
\text { IIAME,IEMAS, } \\
\text { IRES,IGRI }\end{array}$ & $\begin{array}{l}\text { ISO14-9-26 } \\
\text {,IAMB,IINV, } \\
\text { IDSM,ODS, } \\
\text { IEMPV, } \\
\text { IEMAS, } \\
\text { IRES,IGRI }\end{array}$ & $\begin{array}{l}\text { ISO14-9-26, } \\
\text { ISUS,SIPV, } \\
\text { IDSM, } \\
\text { CEMDS, } \\
\text { IIAME, } \\
\text { IEMAS, } \\
\text { IRES,IGRI }\end{array}$ & & $\begin{array}{l}\text { ISO14-9-26, } \\
\text { ISUS,IAMB, } \\
\text { IINV,IDSM, } \\
\text { IIAME,IEMAS }\end{array}$ & & & $\begin{array}{l}\text { ISO14-9-26, } \\
\text { ISUS,SIPV, } \\
\text { IDSM, } \\
\text { CEMDS, } \\
\text { IIAME,IEMAS, } \\
\text { IRES,IGRI }\end{array}$ & ISUS \\
\hline $\begin{array}{l}\text { Impacto } \\
\text { ambiental } \\
\text { por uso de } \\
\text { instalaciones } \\
\text { y equipos }\end{array}$ & & $\begin{array}{l}\text { ISO14-9-26, } \\
\text { IINV,SIPV, } \\
\text { ODS,IEMPV, } \\
\text { IEMAS, IRES }\end{array}$ & & $\begin{array}{l}\text { ISO14-9-26, } \\
\text { SIPV,IDSM, } \\
\text { CEMDS, } \\
\text { IIAME, } \\
\text { IEMAS, } \\
\text { IRES,IGRI }\end{array}$ & IGRI & & & & & $\begin{array}{l}\text { ISO14-9-26, } \\
\text { SIPV,IDSM, } \\
\text { CEMDS, } \\
\text { IIAME, } \\
\text { IEMAS, } \\
\text { IRES,IGRI }\end{array}$ & & $\begin{array}{l}\text { ISO14-9-26, } \\
\text { IDSM,IIAME, } \\
\text { IEMAS }\end{array}$ \\
\hline $\begin{array}{l}\text { Integridad } \\
\text { y procesos } \\
\text { operativos } \\
\text { justos }\end{array}$ & $\begin{array}{l}\text { ISO14-9-26, } \\
\text { IIAME, IGRI }\end{array}$ & $\begin{array}{l}\text { ISUS,IDSM, } \\
\text { CEMDS }\end{array}$ & $\begin{array}{l}\text { ISO14-9-26, } \\
\text { IIAME,IGRI }\end{array}$ & $\begin{array}{l}\text { ISO14-9-26, } \\
\text { IRES,IGRI }\end{array}$ & $\begin{array}{l}\text { ISO14-9-26, } \\
\text { ISUS,SIPV, } \\
\text { IDSM,ODS, } \\
\text { IIAME, } \\
\text { IEMAS,IRES }\end{array}$ & & & $\begin{array}{l}\text { ISO14-9-26, } \\
\text { IRES,IGRI }\end{array}$ & & & & \\
\hline
\end{tabular}




\begin{tabular}{|c|c|c|c|c|c|c|c|c|c|c|c|c|}
\hline 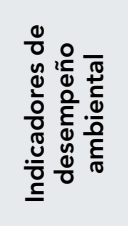 & 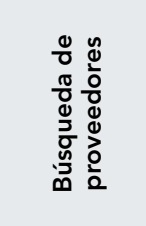 & 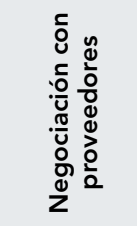 & 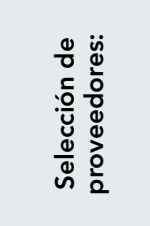 & 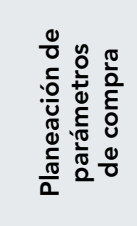 & 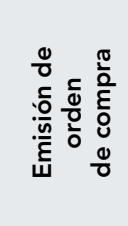 & 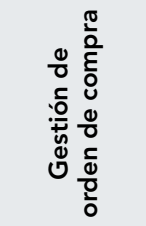 & 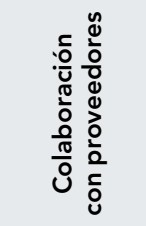 & 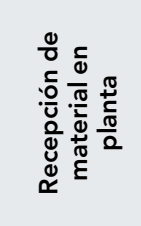 & 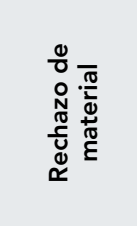 & $\frac{\frac{\mathscr{T}}{\pi}}{\frac{\mathbb{U}}{\mathcal{U}}}$ & 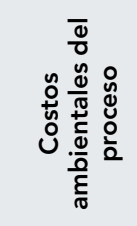 & 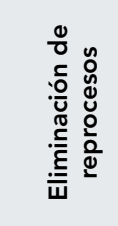 \\
\hline $\begin{array}{l}\text { Material } \\
\text { peligroso } \\
\text { y dañino al } \\
\text { ambiente }\end{array}$ & & & & & & & & $\begin{array}{l}\text { ISUS,SIPV, } \\
\text { IDSM, } \\
\text { CEMDS }\end{array}$ & $\begin{array}{l}\text { ISUS,SIPV, } \\
\text { IDSM, } \\
\text { CEMDS }\end{array}$ & $\begin{array}{l}\text { ISO14-9-26, } \\
\text { ISUS,IEMAS }\end{array}$ & & \\
\hline $\begin{array}{l}\text { Materias } \\
\text { primas sus- } \\
\text { tentables }\end{array}$ & $\begin{array}{l}\text { ISO14-9-26, } \\
\text { ISUS,IDSM, } \\
\text { ODS, IEMAS, } \\
\text { IRES }\end{array}$ & $\begin{array}{l}\text { ISO14-9-26, } \\
\text { ISUS, IAMB, } \\
\text { IINV,SIPV, } \\
\text { IDSM,ODS, } \\
\text { CEMDS, } \\
\text { IIAME, } \\
\text { IEMAS,IGRI }\end{array}$ & $\begin{array}{l}\text { ISO14-9-26, } \\
\text { ISUS,IAMB, } \\
\text { IINV,SIPV, } \\
\text { IDSM, } \\
\text { CEMDS, } \\
\text { IIAME,IEMAS, } \\
\text { IRES,IGRI }\end{array}$ & & & $\begin{array}{l}\text { ISO14-9-26, } \\
\text { ISUS,IAMB, } \\
\text { IINV,SIPV, } \\
\text { IDSM,ODS, } \\
\text { IEMPV,IIAME, } \\
\text { IEMAS,IRES, } \\
\text { IGRI }\end{array}$ & $\begin{array}{l}\text { ISO14-9-26, } \\
\text { ISUS,IAMB, } \\
\text { IINV,SIPV, } \\
\text { IDSM,ODS, } \\
\text { IEMPV,IIAME, } \\
\text { IEMAS,IRES, } \\
\text { IGRI }\end{array}$ & & & & & \\
\hline $\begin{array}{l}\text { Proveedores } \\
\text { con certi- } \\
\text { ficaciones } \\
\text { sustentables }\end{array}$ & $\begin{array}{l}\text { ISO14-9-26, } \\
\text { SIPV,IIAME }\end{array}$ & $\begin{array}{l}\text { ISO14-9-26, } \\
\text { IIAME,IEMAS, } \\
\text { IGRI }\end{array}$ & & & $\begin{array}{l}\text { ISO14-9-26, } \\
\text { IIAME,IRES }\end{array}$ & & $\begin{array}{l}\text { ISO14-9-26, } \\
\text { SIPV,IIAME }\end{array}$ & & & & & $\begin{array}{l}\text { ISO14-9-26, } \\
\text { IIAME,IRES }\end{array}$ \\
\hline $\begin{array}{l}\text { Servicios de } \\
\text { soporte a las } \\
\text { operaciones }\end{array}$ & $\begin{array}{l}\text { ISM,IEMPV, } \\
\text { IGRI }\end{array}$ & $\begin{array}{l}\text { ISO14-9-26, } \\
\text { IINV,SIPV, } \\
\text { ODS,IEMPV, } \\
\text { IEMAS,IRES }\end{array}$ & & IDSM,IGRI & & $\begin{array}{l}\text { ISO14-9-26, } \\
\text { IDSM,IIAME, } \\
\text { IGRI }\end{array}$ & $\begin{array}{l}\text { ISO14-9-26, } \\
\text { ISUS,IDSM, } \\
\text { CEMDS, } \\
\text { IIAME,IEMAS, } \\
\text { IRES }\end{array}$ & ISO14-9-26 & $\begin{array}{l}\text { ISO14-9-26, } \\
\text { IDSM,IIAME, } \\
\text { IGRI }\end{array}$ & & & $\begin{array}{l}\text { ISO14-9-26, } \\
\text { IDSM, } \\
\text { CEMDS, } \\
\text { IEMAS }\end{array}$ \\
\hline $\begin{array}{l}\text { Transferencia } \\
\text { de costos } \\
\text { ambientales }\end{array}$ & & $\begin{array}{l}\text { ISO14-9-26, } \\
\text { IDSM,IEMPV, } \\
\text { IRES,IGRI }\end{array}$ & $\begin{array}{l}\text { ISO14-9-26, } \\
\text { IDSM,ODS, } \\
\text { IEMPV,IGRI }\end{array}$ & $\begin{array}{l}\text { ISO14-9-26, } \\
\text { IDSM,IEMPV, } \\
\text { IRES,IGRI }\end{array}$ & & & $\begin{array}{l}\text { ISO14-9-26, } \\
\text { ISUS,IDSM, } \\
\text { CEMDS, } \\
\text { IIAME, } \\
\text { IEMAS,IRES }\end{array}$ & $\begin{array}{l}\text { ISO14-9-26, } \\
\text { IDSM,IEMPV, } \\
\text { IRES,IGRI }\end{array}$ & $\begin{array}{l}\text { ISO14-9-26, } \\
\text { ISUS,IDSM, } \\
\text { CEMDS, } \\
\text { IEMAS }\end{array}$ & & $\begin{array}{l}\text { ISO14-9-26, } \\
\text { IDSM,ODS, } \\
\text { IEMPV,IGRI }\end{array}$ & \\
\hline $\begin{array}{l}\text { Uso de } \\
\text { materias } \\
\text { primas no } \\
\text { renovables }\end{array}$ & $\begin{array}{l}\text { ISO14-9-26, } \\
\text { ISUS,IAMB, } \\
\text { IDSM,CEMDS, } \\
\text { IGRI }\end{array}$ & & $\begin{array}{l}\text { ISO14-9-26, } \\
\text { IINV,SIPV,ODS, } \\
\text { IEMPV,IEMAS, } \\
\text { IRES }\end{array}$ & $\begin{array}{l}\text { ISO14-9-26, } \\
\text { IINV,IDSM, } \\
\text { IIAME, } \\
\text { IEMAS }\end{array}$ & $\begin{array}{l}\text { ISO14-9-26, } \\
\text { ISUS, } \\
\text { CEMDS, } \\
\text { IEMPV, } \\
\text { IIAME,IRES }\end{array}$ & $\begin{array}{l}\text { ISO14-9-26, } \\
\text { ODS,CEMDS, } \\
\text { IIAME,IEMAS }\end{array}$ & $\begin{array}{l}\text { ISO14-9-26, } \\
\text { ISUS,IDSM, } \\
\text { ODS,IEMAS, } \\
\text { IRES }\end{array}$ & & & & & \\
\hline
\end{tabular}

Fuente: elaboración propia con base a los modelos citados.

Ahora bien, los indicadores son susceptibles de aplicarse de forma general, por etapa del proceso o por resultado de medición del impacto ambiental, solo debe considerarse una perspectiva de alcance de objetivos por resultados en los procesos actuales, dependiendo de los actores del proceso; sea desde la planeación de compra y relación con el proveedor, la entrega de calidad del material y el rechazo de mercancía o el desempeño del personal de abastecimiento; así, desde el enfoque de resultados de impacto ambiental, se definen las actividades con mayor impacto, como el rechazo de material y la eliminación de reprocesos. 


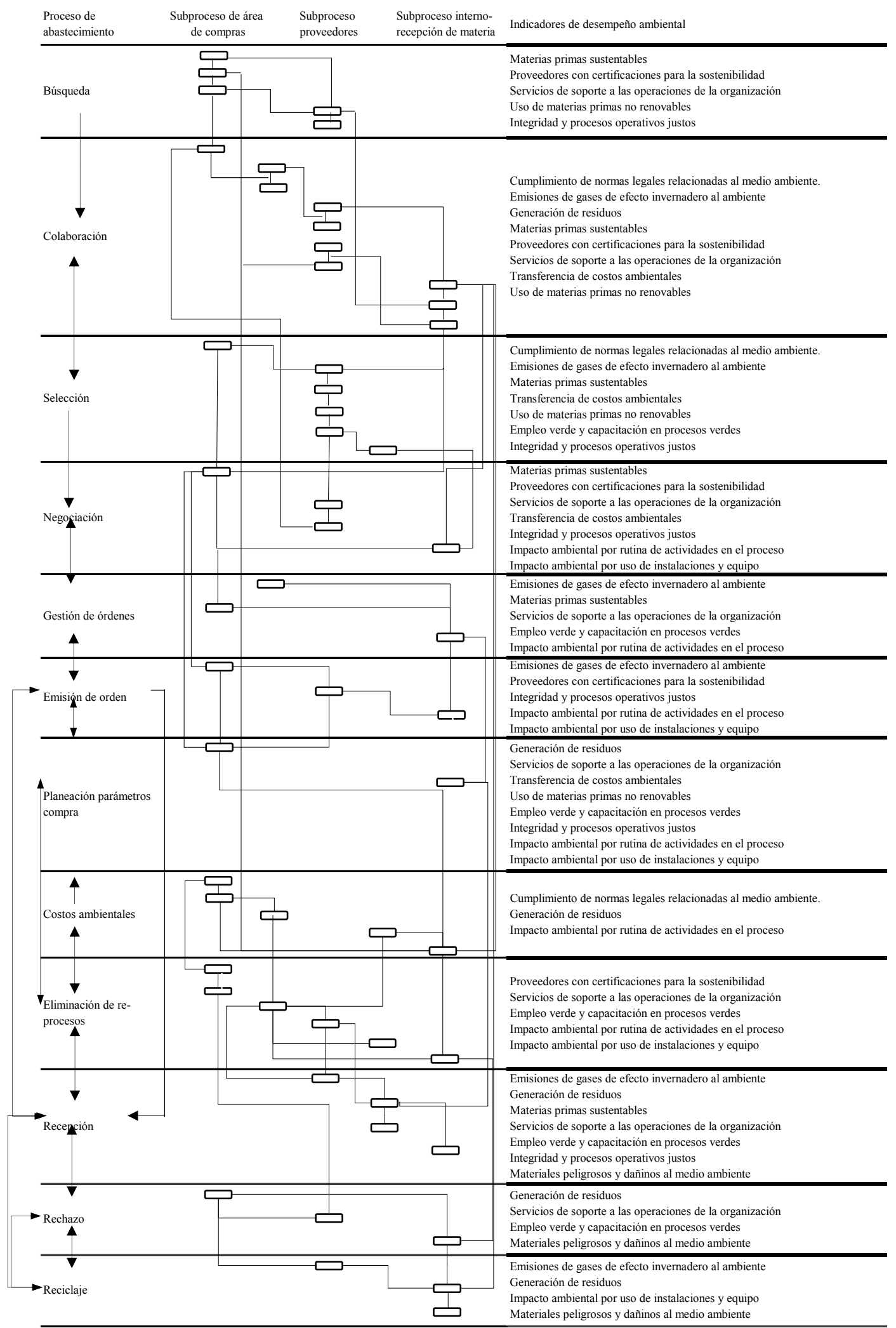

Figura 2. Indicadores de proceso-subproceso de abastecimiento

Fuente: elaboración propia. 


\section{Conclusiones}

La industria del plástico es una de las más importantes en el mundo, sin embargo, conlleva un proceso de transformación energético-intenso, con diversos impactos ambientales que van desde grandes toneladas de emisiones de efecto invernadero hasta la contaminación por la deficiente disposición de sus residuos y reciclaje. Ante ello, y derivado de la presión social de los clientes, estas organizaciones se han visto en la necesidad de establecer, además de las métricas económicas, las de impacto ambiental y han empezado a priorizar la selección de los proveedores más considerados con el medio ambiente, como parte de su cadena de abastecimiento.

Una primera conclusión de la investigación es que los principales indicadores de desempeño ambiental, en el proceso de suministro de la industria mediana del plástico, se relacionan con la generación de emisiones de dióxido de carbono derivados de los residuos generados por el rechazo de material; no obstante que el proveedor gestione la pérdida de inventarios, la generación de residuos sólidos tiende a incrementarse por los retrabajos. Así, la identificación oportuna de errores por rutina de actividades en el proceso es parte crucial para reducir o eliminar su impacto ambiental.

Una segunda conclusión es que resulta un total de 13 indicadores de desempeño ambiental, algunos son coincidentes para uno o más de los tres subprocesos (compras, proveedores y procesos internos), que integran el proceso de abastecimiento; otros son particulares para cada uno de ellos. Así, los indicadores en los proveedores son: cumplimiento de normas legales relacionadas con el medio ambiente, proveedores con certificaciones para la sostenibilidad, generación de residuos, materias primas sustentables, uso de materias primas no renovables, servicios de soporte a la organización y la transferencia de costos ambientales. Mientras que, los indicadores en el subproceso de compra son: empleo verde y capacitación en procesos verde, impacto ambiental por rutina de actividades en el proceso, impacto ambiental por uso de instalaciones y equipos, integridad y procesos operativos justos, servicios de soporte a la organización y generación de residuos. Finalmente, los indicadores asociados al subproceso interno son: emisiones de gases de efecto invernadero al ambiente, generación de residuos, materiales peligrosos y dañinos al ambiente, impacto ambiental por rutina de actividades en el proceso, impacto ambiental por uso 
de instalaciones y equipos, integridad y procesos operativos justos y la transferencia de costos ambientales.

La principal limitante de la investigación fue el escaso número de expertos que participaron en el estudio, esto se debió a la falta de sensibilidad con relación al beneficio real que se obtiene al incorporar indicadores de desempeño ambiental al proceso de suministro.

Este estudio, como un primer acercamiento, busca alentar más investigaciones que permitan consolidar los indicadores propuestos e incluso orientarlos hacia métricas en la huella ecológica para cada fase del proceso de abastecimiento y con ello proporcionar un marco de referencia en la toma de decisiones para mejorar el desempeño ambiental y financiero de una industria menos contaminante.

\section{Referencias}

Appiagyei, A. A., Ayarkwa, J., \& Agyekum, K. (2016). Environmental Considerations in Procurement Decisions: A Literature Review. Sustainability in Environment, 1(1), 40. https://doi.org/10.22158/se.v1n1p40

Asif, M. S., Lau, H., Nakandala, D., Fan, Y., \& Hurriyet, H. (2020, December). Adoption of green supply chain management practices through collaboration approach in developing countries e from literature review to conceptual framework. Journal of Cleaner Production, 276. https://doi.org/10.1016/j.jclepro.2020.124191

Aureli, S., Cardoni, A., Del Baldo, M., \& Lombardi, R. (2018). The balanced scorecard logic in the management control and reporting of small business company networks: A case study. Journal of Accounting and Management Information Systems, 12(2), 191-215. https:// doi.org/10.24818/jamis.2018.02001

Bai, C., \& Satir, A. (2020, July). Barriers for green supplier development programs in manufacturing industry. Resources, Conservation and Recycling, 158. https://doi.org/10.1016/j. resconrec.2020.104756

Balfaqih, H., Nopiah, Z. M., Saibani, N., \& Al-Nory, M. T. (2016). Review of supply chain performance measurement systems: 1998-2015. Computers in Industry, 82, 135-150. https:// doi.org/10.1016/j.compind.2016.07.002

Bolstorff, P., \& Rosenbaum, R. (2011). Supply chain excellence: a handbook for dramatic improvement using the SCOR model. (3. ${ }^{a}$ ed.). AMACOM. 
Borkey, P., \& Leinala, E. (2018, 29-31 May). Considerations and Criteria for Sustainable Plastics from a Chemicals Perspective [Background paper 1]. OECD Global Forum on Environment: Plastics in a Circular Economy. Copenhagen.

Briggs, S. L. K. (2017). Environmental Management Systems for SMEs a Practical Guide for SMEs ISO 14001:2015. ISO.

Cabeza, D. (2014). Logística inversa en la gestión de la cadena de suministro (2. ${ }^{\mathrm{a}}$ ed.). Alfaomega.

Calderón, J. F. (2012). El Concepto de Responsabilidad Social Empresarial. https://www.cemefi.org/esr/images/stories/pdf/esr/concepto_esr.pdf

Carter, C. R., \& Rogers, D. S. (2008). A framework of sustainable supply chain management: Moving toward new theory. International Journal of Physical Distribution and Logistics Management, 38(5), 360-387. https://doi.org/10.1108/09600030810882816

Castelan, G. (2018). LIFE CYCLE THINKING. How LCA can belp reducing plasticsmarine litter a knowledgeable and efficient way: managing is measuring. Plastics Europe.

CEPAL. (2018). La Unión Europea y América Latina y el Caribe: Estrategias convergentes y sostenibles ante la coyuntura global. https://www.cepal.org/es/publicaciones/43740-la-union-europea-america-latina-caribe-estrategias-convergentes-sostenibles-la

Cervera-Ferri, J. L., \& Ureña, M. L. (2017). Indicadores de producción verde: una guía para avanzar hacia el desarrollo sostenible. CEPAL. https://www.cepal.org/es/publicaciones/ 40967-indicadores-produccion-verde-guia-avanzar-desarrollo-sostenible

Chávez, M. J. (2005). Coordinación de políticas públicas para el desarrollo sostenible del sector turismo en el Perú [Serie Medio ambiente y desarrollo]. CEPAL. https://repositorio.cepal. org/bitstream/handle/11362/5653/1/S05937_es.pdf

Chopra, S., \& Meindl, P. (2013). Administración de la cadena de suministro. Estrategia, planeación y operación (5. ${ }^{\mathrm{a}}$ ed.). Pearson Educación.

Crespin-Mazet, F., \& Dontenwill, E. (2012, December). Sustainable procurement: Building legitimacy in the supply network. Journal of Purchasing and Supply Management, 18(4), 207-217. https://doi.org/10.1016/j.pursup.2012.01.002

Dini, M., \& Stumpo, G. (2011). Políticas para la innovación en las pequeñas y medianas empresas en América Latina [Documentos de proyectos e investigación]. CEPAL. https:// repositorio.cepal.org/handle/11362/3868

Dragomir, V. D. (2018, September). How do we measure corporate environmental performance? A critical review. Journal of Cleaner Production, 196, 1124-1157. https://doi. org/10.1016/j.jclepro.2018.06.014 
EMAs. (2013). Guía Práctica para la aplicación del Reglamento eMAs. https://www.comunidad.madrid/sites/default/files/doc/medio-ambiente/emas_reglamento_guia_practica_ rev_2013.pdf

Farooque, M., Zhang, A., Thürer, M., Qu, T., \& Huisingh, D. (2019, August). Circular supply chain management: A definition and structured literature review. Journal of Cleaner Production, 228, 882-900. https://doi.org/10.1016/j.jclepro.2019.04.303

Forkmann, S., Henneberg, S. C., Naudé, P., \& Mitrega, M. (2016, August). Supplier relationship management capability: a qualification and extension. Industrial Marketing Management, 57, 185-200. https://doi.org/10.1016/j.indmarman.2016.02.003

Ganguly, K., \& Shankar Rai, S. (2018). Evaluating the key performance indicator for supply chain information system implementation using IPA model. Benchmarking: An International Journal, 25(6), 1844-1863. https://doi.org/10.1108/BIJ-03-2017-0041

Giannakis, M., Dubey, R., Vlachos, I., \& Ju, Y. (2020, febrero). Supplier sustainability performance evaluation using the analytic network process. Journal of Cleaner Production, 247. https://doi.org/10.1016/j.jclepro.2019.119439

Giosa Zuazua, N., \& Fernández Massi, M. (2017). La competitividad de las empresas de productos plásticos: una tipología a partir de factores empresariales y estructurales. En La Encuesta Nacional de Dinámica de Empleo e Innovación (ENDEI) como herramienta de análisis La innovación y el empleo en la industria manufacturera argentina (pp. 279301). CEPAL.

Giunipero, L. C., Hooker, R. E., \& Denslow, D. (2012, december). Purchasing and supply management sustainability: Drivers and barriers. Journal of Purchasing and Supply Management, 18(4), 258-269. https://doi.org/10.1016/j.pursup.2012.06.003

Global Sustainability Standards Board (GSsB). (2016a). Gri 201: Desempeño económico 2016. https://www.globalreporting.org/standards/media/1442/spanish-gri-201-economic-performance-2016.pdf

Global Sustainability Standards Board (GSSB). (2016b). Gri 204: Prácticas de adquisición 2016. https://economistas.es/Contenido/REA/EINF-GRI/GRI\%20204-1.pdf

Global Sustainability Standards Board (GSSB). (2016c). Gri 301: Materiales 2016. https://www. globalreporting.org/standards/media/1448/spanish-gri-301-materials-2016.pdf

Global Sustainability Standards Board (GssB). (2016d). Gri 302: Energía 2016. https://www. globalreporting.org/standards/media/1449/spanish-gri-302-energy-2016.pdf

Global Sustainability Standards Board (GSSB). (2016e). Gri 306: Efluentes y residuos 2016. https://www.globalreporting.org/standards/media/1453/spanish-gri-306-effluents-andwaste-2016.pdf 
Global Sustainability Standards Board (GSsB). (2016f). Gri 308: Evaluación ambiental de proveedores 2016. https://www.globalreporting.org/standards/media/1455/spanish-gri-308-supplier-environmental-assessment-2016.pdf

Global Sustainability Standards Board (GssB). (2016g). Gri 402: Relaciones trabajador - empresa. https://www.globalreporting.org/standards/media/1457/spanish-gri-402-labormanagement-relations-2016.pdf

Global Sustainability Standards Board (GSsB). (2016h). Gri 404: formación y enseñanza 2016. https://www.globalreporting.org/standards/media/1459/spanish-gri-404-training-and-education-2016.pdf

Global Sustainability Standards Board (GSSB). (2016i). Gri 414: Evaluación social de los proveedores 2016. https://www.globalreporting.org/standards/media/1469/spanish-gri-414-supplier-social-assessment-2016.pdf

Global Sustainability Standards Board (GssB). (2016j). GRI 406: No discriminación. https:// www.globalreporting.org/standards/media/1461/spanish-gri-406-non-discrimination-2016. pdf

Graça, P., \& Camarinha-Matos, L. M. (2017, March). Performance indicators for collaborative business ecosystems - Literature review and trends. Technological Forecasting and Social Change, 116, 237-255. https://doi.org/10.1016/j.techfore.2016.10.012

Hoejmose, S. U., \& Adrien-Kirby, A. J. (2012, dicembre). Socially and environmentally responsible procurement: A literature review and future research agenda of a managerial issue in the 21st century. Journal of Purchasing and Supply Management, 18(4), 232-242. https:// doi.org/10.1016/j.pursup.2012.06.002

Horvath, B., Mallinguh, E., \& Fogarassy, C. (2018). Designing Business Solutions for plastic waste management to enhance circular transitions in Kenya. Sustainability, 10(5). https:// doi.org/10.3390/su10051664

IHOBE. (1999). Guía de Indicadores Medioambientales Para La Empresa. https://alojamientos. uva.es/guia_docente/uploads/2013/430/52300/1/Documento.pdf

Instituto Nacional de Estadística, Geografía e Informática. (1999). Indicadores de Desarrollo Sustentable en México. http://www.nies.go.jp/db/sdidoc/indicadores_desarrollo_sustentable.pdf

IsO. (2015). Iso 9001:2015(es) Quality management systems - Requirements. https://www.iso. org/obp/ui/\#iso:std:iso:9001:ed-5:v1:es

ISO. (2015a). Introduction to Iso 14001:2015. https://www.iso.org/files/live/sites/isoorg/files/ store/en/PUB100371.pdf

ISO. (2015b). ISo 14001:2015(es). https://www.iso.org/obp/ui\#iso:std:iso:14001:ed-3:v1:es 
Iso. (2016). Iso 9001:2015 for Small Enterprises What to do? https://www.iso.org/publication/ PUB100406.html

ISO. (2017). ISO 20400:2017(E) Sustainable procurement - Guidance. https://www.iso.org/ standard/63026.html

Kalubanga, M. (2012). Sustainable procurement: Concept, and practical implications for the procurement process. International Journal of Economics and Management Sciences, 1(7), 1-7. https://www.researchgate.net/publication/281175084_SUSTAINABLE_PROCUREMENT_ Concept_and_Practical_Implications_for_the_Procurement_Process

Kipp, A., Jiang, T., Fugini, M., \& Salomie, I. (2012, February). Layered Green Performance Indicators. Future Generation Computer Systems, 28(2), 478-489. https://doi.org/10.1016/j. future.2011.05.005

Krajewski, L. J., Ritzman, L., \& Malhotra, M. (2013). Administración de Operaciones. Procesos $y$ cadena de suministro (10. ${ }^{\mathrm{a}}$ ed.). Pearson Educación.

Lahane, S., Kant, R., \& Shankar, R. (2020, June). Circular supply chain management: A stateof-art review and future opportunities. Journal of Cleaner Production, 258. https://doi. org/10.1016/j.jclepro.2020.120859

López Blanco, J., \& Rodríguez Gamiño, M. de L. (2009). Desarrollo de indicadores ambientales $y$ de sustentabilidad en México. Instituto de Geografía.

López López, V. M. (2017). Sustentabilidad y desarrollo sustentable Origen, precisiones conceptuales y metodología operativa (2. ${ }^{\mathrm{a}}$ ed.). Trillas.

Malviya, R. K., \& Kant, R. (2017). Modeling the enablers of green supply chain management: An integrated ISM - fuzzy MICMAC approach. Benchmarking: An International Journal, 24(2), 536-568. https://www.emerald.com/insight/content/doi/10.1108/BIJ-08-2015-0082/ full/html

Manteiga, L. (2000). Los indicadores ambientales como instrumento para el desarrollo de la política ambiental y su integración de otras políticas. Estadística y Medio Ambiente, 75-87.

Martínez, R. (2009). Guía metodológica para desarrollar indicadores ambientales y de desarrollo sostenible en países de América Latina y el Caribe. Naciones Unidas; CEPAL. https:// repositorio.cepal.org/handle/11362/5502

Mathiyazhagan, K., Haq, A. N., \& Baxi, V. (2016). Analysing the barriers for the adoption of green supply chain management - The Indian plastic industry perspective. International Journal of Business Performance and Supply Chain Modelling, 8(1), 46-65. https://doi. org/10.1504/IJBPSCM.2016.076000 
Meherishi, L., Narayana, S. A., \& Ranjani, K. S. (2019, November). Sustainable packaging for supply chain management in the circular economy: A review. Journal of Cleaner Production, 237. https://doi.org/10.1016/j.jclepro.2019.07.057

Mercado, S. (2002). Compras Principios y Aplicaciones (4. $\left.{ }^{\mathrm{a}} \mathrm{ed}.\right)$. Limusa Noriega.

Mercado, S. (2009). Administración de pequeñas y medianas empresas Estrategias de crecimiento (1. ${ }^{\mathrm{a}}$ ed.). Litográfica Ingramex.

Mura, M., Longo, M., Micheli, P., \& Bolzani, D. (2018). The Evolution of Sustainability Measurement Research. International Journal of Management Reviews, 20(3), 661-695. https://doi.org/10.1111/ijmr.12179

OECD. (2018). Improving plastics management: trends, policy responses, and the role of international co-operation and trade. Environmental Policy Paper (12), 12-20. https://doi. org/10.1787/c5f7c448-en

огт. (2018). El Programa Empleos Verdes de la оIт. https://www.ilo.org/wcmsp5/groups/public/---ed_emp/---emp_ent/documents/publication/wcms_432895.pdf

ONU. (2017). Informe de los Objetivos de Desarrollo Sostenible 2017. https://unstats.un.org/ sdgs/files/report/2017/thesustainabledevelopmentgoalsreport2017_spanish.pdf

Paesbrugghe, B., Rangarajan, D., Sharma, A., Syam, N., \& Jha, S. (2017, April). Purchasing-driven sales: Matching sales strategies to the evolution of the purchasing function. Industrial Marketing Management, 62, 171-184. https://doi.org/10.1016/j.indmarman.2016.09.002

Rivera González, I. A., Cristóbal Vázquez, I. M., \& Aguilar Fernández, M. (2011). Administración de la cadena de suministros. Diseño y aplicación de modelos para empresas mexicanas (1. ${ }^{a}$ ed.). Instituto Politécnico Nacional.

Ruiz Torres, A. J., Ablanedo Rosas, J. H., \& Ayala Cruz, J. (2013). Modelo de asignación de compras a proveedores considerando su flexibilidad y probabilidad de incumplimiento en la entrega. Estudios Gerenciales, 28(122), 29-48. https://doi.org/10.1016/s01235923(12)70192-4

Samaniego, J., \& Schneider, H. (2010). La huella del carbono en la producción, distribución y consumo de bienes y servicios. CEPAL.

Sánchez López, O. R., \& Meza Arístigue, L. (2010, 27-30 de abril). Indicadores básicos para diagnosticar la responsabilidad social empresarial. XIV Congreso Internacional de la Academia de Ciencias Administrativas A. C. (ACACIA) (pp. 664-682). Escuela de Graduados en Administración y Dirección de Empresas del Tecnológico de Monterrey, N. L. http:// acacia.org.mx/busqueda/pdf/INDICADORES_B_SICOS.pdf 
Schneider, L., \& Wallenburg, C. M. (2012). Implementing sustainable sourcing-Does purchasing need to change? Journal of Purchasing and Supply Management, 18(4), 243-257. https:// doi.org/10.1016/j.pursup.2012.03.002

Sönnichsen, S. D., \& Clement, J. (2020, February). Review of green and sustainable public procurement: Towards circular public procurement. Journal of Cleaner Production, 245. https://doi.org/10.1016/j.jclepro.2019.118901

Sundarakani, B., Goh, M., De Souza, R., \& Shun, C. (2008). Measuring carbon footprints across the supply chain. 13th International Symposium on Logistics (ISL2008): Integrating the Global Supply Chain (pp. 555-562). Nottingham University Business School, UK.

Walker, H., Miemczyk, J., Johnsen, T., \& Spencer, R. (2012, December). Sustainable procurement: Past, present and future. Journal of Purchasing and Supply Management, 18(4), 201-206. https://doi.org/10.1016/j.pursup.2012.11.003 


\section{Anexo 1. Indicadores de desempeño ambiental por modelo}

\begin{tabular}{|c|c|c|c|c|c|c|c|c|c|c|c|c|c|c|c|}
\hline \multirow{2}{*}{$\begin{array}{l}\text { Indicadores de } \\
\text { desempeño } \\
\text { ambiental }\end{array}$} & \multirow{2}{*}{$\begin{array}{c}\text { Factor } \\
\text { ambiental } \\
\text { (medición) }\end{array}$} & \multicolumn{13}{|c|}{ Modelos } & \multirow[b]{2}{*}{ Total } \\
\hline & & $\begin{array}{c}\text { Iso } \\
14-9-26\end{array}$ & ISUS & IAMB & IINV & SIPV & IDSM & ODS & CEMDS & IEMPV & IIAME & IEMAS & IRES & IGRI & \\
\hline $\begin{array}{l}\text { Empleo Verde y } \\
\text { Capacitación en } \\
\text { procesos verde }\end{array}$ & $\begin{array}{l}\text { Empleo Verde y } \\
\text { Capacitación en } \\
\text { procesos verde }\end{array}$ & $x$ & $x$ & $x$ & $x$ & $x$ & $x$ & $x$ & & $x$ & $x$ & $x$ & $x$ & $x$ & 12 \\
\hline $\begin{array}{l}\text { Generación de } \\
\text { residuos }\end{array}$ & $\begin{array}{l}\text { Contaminación } \\
\text { de residuos de } \\
\text { procesos }\end{array}$ & $x$ & $x$ & $x$ & $x$ & $x$ & $x$ & & $x$ & & $x$ & $x$ & $x$ & $x$ & 11 \\
\hline $\begin{array}{l}\text { Materias primas } \\
\text { sustentables }\end{array}$ & $\begin{array}{l}\text { Consumo de } \\
\text { agua }\end{array}$ & $x$ & $x$ & $x$ & $x$ & $x$ & $x$ & $x$ & $x$ & & $x$ & $x$ & & $x$ & 11 \\
\hline $\begin{array}{l}\text { Uso de materias pri- } \\
\text { mas no renovables }\end{array}$ & $\begin{array}{l}\text { Cambios en } \\
\text { el paisaje por } \\
\text { procesos }\end{array}$ & $x$ & $x$ & $x$ & $x$ & $x$ & $x$ & $x$ & & & $x$ & $x$ & $x$ & $x$ & 11 \\
\hline $\begin{array}{l}\text { Uso de materias pri- } \\
\text { mas no renovables }\end{array}$ & $\begin{array}{l}\text { Materias primas } \\
\text { sustentables }\end{array}$ & $x$ & $x$ & $x$ & $x$ & $x$ & $x$ & & $x$ & $x$ & $x$ & $x$ & & $x$ & 11 \\
\hline $\begin{array}{l}\text { Cumplimiento de } \\
\text { normas legales } \\
\text { relacionadas con el } \\
\text { medio ambiente. } \\
\text { Proveedores con } \\
\text { certificaciones para } \\
\text { la sostenibilidad }\end{array}$ & Certificaciones & $x$ & $x$ & & & $x$ & $x$ & & $x$ & & $x$ & $x$ & $x$ & $x$ & 9 \\
\hline $\begin{array}{l}\text { Cumplimiento de } \\
\text { normas legales } \\
\text { relacionadas con el } \\
\text { medio ambiente. } \\
\text { Proveedores con } \\
\text { certificaciones para } \\
\text { la sostenibilidad }\end{array}$ & $\begin{array}{l}\text { Cumplimiento } \\
\text { de normas } \\
\text { legales medio } \\
\text { ambientales, } \\
\text { certificaciones y } \\
\text { políticas guber- } \\
\text { namentales }\end{array}$ & $x$ & $x$ & & & $x$ & $x$ & & $x$ & & $x$ & $x$ & $x$ & $x$ & 9 \\
\hline $\begin{array}{l}\text { Empleo Verde y } \\
\text { Capacitación en } \\
\text { procesos verde }\end{array}$ & $\begin{array}{l}\text { Protección } \\
\text { del personal y } \\
\text { desempeño }\end{array}$ & $x$ & & $x$ & $x$ & & $x$ & $x$ & & $x$ & & $x$ & $x$ & $x$ & 9 \\
\hline $\begin{array}{l}\text { Uso de materias pri- } \\
\text { mas no renovables }\end{array}$ & $\begin{array}{l}\text { Uso de agua, } \\
\text { suelo, árboles, } \\
\text { minerales, etc. }\end{array}$ & $x$ & & $x$ & $x$ & & $x$ & & $x$ & $x$ & & $x$ & $x$ & $x$ & 9 \\
\hline $\begin{array}{l}\text { Uso de materias pri- } \\
\text { mas no renovables }\end{array}$ & $\begin{array}{l}\text { Servicios } \\
\text { prestados a la } \\
\text { organización }\end{array}$ & $x$ & $x$ & & & & & $x$ & $x$ & $x$ & $x$ & $x$ & $x$ & $x$ & 9 \\
\hline $\begin{array}{l}\text { Generación de } \\
\text { residuos }\end{array}$ & $\begin{array}{l}\text { Reciclado de re- } \\
\text { siduos, reproce- } \\
\text { sos de residuos, } \\
\text { eliminación de } \\
\text { residuos. }\end{array}$ & $x$ & $x$ & $x$ & & & $x$ & & $x$ & & & $x$ & $x$ & $x$ & 8 \\
\hline $\begin{array}{l}\text { Generación de } \\
\text { residuos }\end{array}$ & $\begin{array}{l}\text { Uso de equipo } \\
\text { de trabajo }\end{array}$ & $x$ & $x$ & & & $x$ & $x$ & $x$ & & & $x$ & $x$ & $x$ & & 8 \\
\hline $\begin{array}{l}\text { Materias primas } \\
\text { sustentables }\end{array}$ & $\begin{array}{l}\text { Consumo de } \\
\text { energía eléc- } \\
\text { trica }\end{array}$ & $x$ & $x$ & $x$ & & $x$ & $x$ & $x$ & & & $x$ & $x$ & & & 8 \\
\hline
\end{tabular}




\begin{tabular}{|c|c|c|c|c|c|c|c|c|c|c|c|c|c|c|c|}
\hline \multirow{2}{*}{$\begin{array}{c}\text { Indicadores de } \\
\text { desempeño } \\
\text { ambiental }\end{array}$} & \multirow{2}{*}{$\begin{array}{c}\text { Factor } \\
\text { ambiental } \\
\text { (medición) }\end{array}$} & \multicolumn{13}{|c|}{ Modelos } & \multirow[b]{2}{*}{ Total } \\
\hline & & $\begin{array}{c}\text { ISO } \\
14-9-26\end{array}$ & ISUS & IAMB & IINV & SIPV & IDSM & ODS & CEMDS & IEMPV & IIAME & IEMAS & IRES & IGRI & \\
\hline $\begin{array}{l}\text { Materias primas } \\
\text { sustentables }\end{array}$ & $\begin{array}{l}\text { Contaminación } \\
\text { atmosférica: } \\
\text { emisiones de } \\
\text { gases de efecto } \\
\text { invernadero por } \\
\text { persona }\end{array}$ & $x$ & $x$ & $x$ & & & $x$ & & $x$ & $x$ & $x$ & & $x$ & & 8 \\
\hline $\begin{array}{l}\text { Transferencia de } \\
\text { costos ambientales }\end{array}$ & $\begin{array}{l}\text { Costos medio } \\
\text { ambientales y } \\
\text { transferencia de } \\
\text { costos ambien- } \\
\text { tales }\end{array}$ & $x$ & & & & $x$ & $x$ & & $x$ & & $x$ & $x$ & $x$ & $x$ & 8 \\
\hline $\begin{array}{l}\text { Transferencia de } \\
\text { costos ambientales }\end{array}$ & $\begin{array}{l}\text { Inversión en } \\
\text { protección } \\
\text { ambiental, } \\
\text { presupuesto } \\
\text { que se destina } \\
\text { para el medio } \\
\text { ambiente }\end{array}$ & $x$ & $x$ & & & $x$ & $x$ & $x$ & $x$ & & & & $x$ & $x$ & 8 \\
\hline $\begin{array}{l}\text { Cumplimiento de } \\
\text { normas legales } \\
\text { relacionadas con el } \\
\text { medio ambiente. } \\
\text { Proveedores con } \\
\text { certificaciones para } \\
\text { la sostenibilidad }\end{array}$ & $\begin{array}{l}\text { Ruido, olor, } \\
\text { polvo, tráfico }\end{array}$ & $x$ & $x$ & & & & $x$ & & $x$ & & $x$ & $x$ & $x$ & & 7 \\
\hline $\begin{array}{l}\text { Empleo Verde y } \\
\text { Capacitación en } \\
\text { procesos verde }\end{array}$ & $\begin{array}{l}\text { Tecnologías } \\
\text { verdes }\end{array}$ & $x$ & & & $x$ & $x$ & & $x$ & & $x$ & & $x$ & $x$ & & 7 \\
\hline $\begin{array}{l}\text { Materias primas } \\
\text { sustentables }\end{array}$ & $\begin{array}{l}\text { Degradación } \\
\text { del suelo }\end{array}$ & $x$ & $x$ & $x$ & $x$ & & $x$ & & & & $x$ & $x$ & & & 7 \\
\hline $\begin{array}{l}\text { Generación de } \\
\text { residuos }\end{array}$ & $\begin{array}{l}\text { Impacto am- } \\
\text { biental por in- } \\
\text { ventarios, rutina } \\
\text { de actividades, } \\
\text { servicio en el } \\
\text { proceso. }\end{array}$ & $x$ & $x$ & & & & $x$ & $x$ & & & & $x$ & $x$ & & 6 \\
\hline $\begin{array}{l}\text { Materiales peligrosos } \\
\text { y dañinos al am- } \\
\text { biente }\end{array}$ & $\begin{array}{l}\text { Uso de materia- } \\
\text { les químicos y } \\
\text { peligrosos }\end{array}$ & $x$ & $x$ & $x$ & & & $x$ & & $x$ & & & & & $x$ & 6 \\
\hline $\begin{array}{l}\text { Materias primas } \\
\text { sustentables }\end{array}$ & $\begin{array}{l}\text { Consumo de } \\
\text { combustibles: }\end{array}$ & $x$ & $x$ & & & & & & $x$ & & $x$ & $x$ & $x$ & & 6 \\
\hline $\begin{array}{l}\text { Empleo Verde y } \\
\text { Capacitación en } \\
\text { procesos verde }\end{array}$ & $\begin{array}{l}\text { Capacitación y } \\
\text { concientización } \\
\text { en procesos } \\
\text { productivos }\end{array}$ & $x$ & & & & & $x$ & & & $x$ & & & $x$ & $x$ & 5 \\
\hline $\begin{array}{l}\text { Impacto ambiental } \\
\text { por rutina de activi- } \\
\text { dades en el proceso. } \\
\text { Impacto ambiental } \\
\text { por uso de instalacio- } \\
\text { nes y equipos }\end{array}$ & $\begin{array}{l}\text { Integración } \\
\text { del ambiente } \\
\text { y el desarrollo } \\
\text { en la toma de } \\
\text { decisiones }\end{array}$ & $x$ & & & & & $x$ & $x$ & & $x$ & & & & $x$ & 5 \\
\hline $\begin{array}{l}\text { Materias primas } \\
\text { sustentables }\end{array}$ & $\begin{array}{l}\text { Destrucción de } \\
\text { flora }\end{array}$ & $x$ & & & $x$ & & $x$ & & & & $x$ & $x$ & & & 5 \\
\hline $\begin{array}{l}\text { Transferencia de } \\
\text { costos ambientales }\end{array}$ & $\begin{array}{l}\text { Pérdida por } \\
\text { desastres natu- } \\
\text { rales }\end{array}$ & $x$ & $x$ & & & & $x$ & & $x$ & & & $x$ & & & 5 \\
\hline
\end{tabular}




\begin{tabular}{|c|c|c|c|c|c|c|c|c|c|c|c|c|c|c|c|}
\hline \multirow{2}{*}{$\begin{array}{c}\text { Indicadores de } \\
\text { desempeño } \\
\text { ambiental }\end{array}$} & \multirow{2}{*}{$\begin{array}{c}\text { Factor } \\
\text { ambiental } \\
\text { (medición) }\end{array}$} & \multicolumn{13}{|c|}{ Modelos } & \multirow[b]{2}{*}{ Tota } \\
\hline & & $\begin{array}{c}150 \\
14-9-26\end{array}$ & ISUS & IAMB & IINV & SIPV & IDSM & ODS & CEMDS & IEMPV & IIAME & IEMAS & IRES & IGRI & \\
\hline $\begin{array}{l}\text { Uso de materias pri- } \\
\text { mas no renovables }\end{array}$ & $\begin{array}{l}\text { Patrón y } \\
\text { cambios de } \\
\text { consumo }\end{array}$ & $x$ & & & & & & $x$ & $x$ & & $x$ & $x$ & & & 5 \\
\hline $\begin{array}{l}\text { Cumplimiento de } \\
\text { normas legales } \\
\text { relacionadas con el } \\
\text { medio ambiente. } \\
\text { Proveedores con } \\
\text { certificaciones para } \\
\text { la sostenibilidad }\end{array}$ & $\begin{array}{l}\text { Responsabilidad } \\
\text { social institu- } \\
\text { cional y dona- } \\
\text { ciones }\end{array}$ & & $x$ & & & $x$ & $x$ & & $x$ & & & & & & 4 \\
\hline $\begin{array}{l}\text { Generación de } \\
\text { residuos }\end{array}$ & Conformidad & $x$ & & & & & $x$ & & & & $x$ & & & $x$ & 4 \\
\hline $\begin{array}{l}\text { Materias primas } \\
\text { sustentables }\end{array}$ & $\begin{array}{l}\text { Destrucción de } \\
\text { fauna }\end{array}$ & $x$ & & & & & $x$ & & & & $x$ & $x$ & & & 4 \\
\hline $\begin{array}{l}\text { Materias primas } \\
\text { sustentables }\end{array}$ & $\begin{array}{l}\text { Uso de combus- } \\
\text { tibles fósiles }\end{array}$ & $x$ & & & & & $x$ & & $x$ & & & $x$ & & & 4 \\
\hline $\begin{array}{l}\text { Uso de materias pri- } \\
\text { mas no renovables }\end{array}$ & Productos & $x$ & & & & & & & & & $x$ & $x$ & & $x$ & 4 \\
\hline $\begin{array}{l}\text { Generación de } \\
\text { residuos }\end{array}$ & $\begin{array}{l}\text { Ingresos, gastos } \\
\text { corrientes e } \\
\text { inversiones }\end{array}$ & $x$ & & & & $x$ & & & & & $x$ & & & & 3 \\
\hline $\begin{array}{l}\text { Generación de } \\
\text { residuos }\end{array}$ & $\begin{array}{l}\text { Respuesta a } \\
\text { incidentes de } \\
\text { impacto am- } \\
\text { biental }\end{array}$ & $x$ & & & & & & & & & $x$ & & $x$ & & 3 \\
\hline $\begin{array}{l}\text { Integridad y proce- } \\
\text { sos operativos justos }\end{array}$ & Corrupción & $x$ & & & & & & & & & & & $x$ & $x$ & 3 \\
\hline $\begin{array}{l}\text { Integridad y proce- } \\
\text { sos operativos justos }\end{array}$ & $\begin{array}{l}\text { Reporte y divul- } \\
\text { gación }\end{array}$ & & & & & & $x$ & & & $x$ & & & & $x$ & 3 \\
\hline $\begin{array}{l}\text { Materiales peligrosos } \\
\text { y dañinos al am- } \\
\text { biente }\end{array}$ & $\begin{array}{l}\text { Identificación } \\
\text { de contamina- } \\
\text { ción por resi- } \\
\text { duos dañinos } \\
\text { en el ambiente }\end{array}$ & & $x$ & & & & $x$ & & $x$ & & & & & & 3 \\
\hline $\begin{array}{l}\text { Materias primas } \\
\text { sustentables }\end{array}$ & $\begin{array}{l}\text { Uso de trans- } \\
\text { porte para la } \\
\text { organización }\end{array}$ & $x$ & $x$ & & & & & & & & & $x$ & & & 3 \\
\hline $\begin{array}{l}\text { Servicios de soporte } \\
\text { a las operaciones de } \\
\text { la organización }\end{array}$ & $\begin{array}{l}\text { Suministro y } \\
\text { entrega }\end{array}$ & $x$ & & & & & & & & & $x$ & & & $x$ & 3 \\
\hline $\begin{array}{l}\text { Transferencia de } \\
\text { costos ambientales }\end{array}$ & $\begin{array}{l}\text { Balance } \\
\text { Financiero } \\
\text { ambiental } \\
\text { (inversiones } \\
\text { y gastos para } \\
\text { la protección } \\
\text { ambiental) } \\
\end{array}$ & $x$ & & & & & & & & & & & $x$ & $x$ & 3 \\
\hline $\begin{array}{l}\text { Generación de } \\
\text { residuos }\end{array}$ & $\begin{array}{l}\text { Ingresos por } \\
\text { residuos o des- } \\
\text { perdicios }\end{array}$ & $x$ & & & & $x$ & & & & & & & & & 2 \\
\hline $\begin{array}{l}\text { Generación de } \\
\text { residuos }\end{array}$ & $\begin{array}{l}\text { Grado de con- } \\
\text { formidad de los } \\
\text { proveedores de } \\
\text { servicios }\end{array}$ & $x$ & & & & & & & & & $x$ & & & & 2 \\
\hline
\end{tabular}




\begin{tabular}{|c|c|c|c|c|c|c|c|c|c|c|c|c|c|c|c|}
\hline \multirow{2}{*}{$\begin{array}{c}\text { Indicadores de } \\
\text { desempeño } \\
\text { ambiental }\end{array}$} & \multirow{2}{*}{$\begin{array}{c}\text { Factor } \\
\text { ambiental } \\
\text { (medición) }\end{array}$} & \multicolumn{13}{|c|}{ Modelos } & \multirow[b]{2}{*}{ Tota } \\
\hline & & $\begin{array}{c}\text { ISO } \\
14-9-26\end{array}$ & ISUS & IAMB & IINV & SIPV & IDSM & ODS & CEMDS & IEMPV & IIAME & IEMAS & IRES & IGRI & \\
\hline $\begin{array}{l}\text { Generación de } \\
\text { residuos }\end{array}$ & $\begin{array}{l}\text { Impacto para la } \\
\text { obtención de la } \\
\text { materia prima }\end{array}$ & $x$ & & & & & & & & & & & & $x$ & 2 \\
\hline $\begin{array}{l}\text { Integridad y proce- } \\
\text { sos operativos justos }\end{array}$ & $\begin{array}{l}\text { Libertad de } \\
\text { asociación }\end{array}$ & & & & & & $x$ & & & & & & & $x$ & 2 \\
\hline $\begin{array}{l}\text { Materias primas } \\
\text { sustentables }\end{array}$ & $\begin{array}{l}\text { Cambio climáti- } \\
\text { co y energía }\end{array}$ & & $x$ & & & & & & & & & & & $x$ & 2 \\
\hline $\begin{array}{l}\text { Integridad y proce- } \\
\text { sos operativos justos }\end{array}$ & $\begin{array}{l}\text { Transparencia e } \\
\text { integridad }\end{array}$ & & & & & & & & & & & & & $x$ & 1 \\
\hline $\begin{array}{l}\text { Materiales peligrosos } \\
\text { y dañinos al am- } \\
\text { biente }\end{array}$ & $\begin{array}{l}\text { Uso de materia- } \\
\text { les peligrosos }\end{array}$ & & $x$ & & & & & & & & & & & & 1 \\
\hline $\begin{array}{l}\text { Servicios de soporte } \\
\text { a las operaciones de } \\
\text { la organización }\end{array}$ & $\begin{array}{l}\text { Atención a } \\
\text { clientes }\end{array}$ & & & & & & & & & & & & & $x$ & 1 \\
\hline
\end{tabular}

Fuente: elaboración propia a partir de los modelos citados.

Nota: el factor de medición es el nombre del indicador tal como aparece en el modelo. No obstante, se reagruparon en categorías más amplias, pero son representativas del concepto al que aluden. 